Document downloaded from:

http://hdl.handle.net/10251/58346

This paper must be cited as:

Vicente Quiles, CP.; Cogollos Borras, S.; Boukari, B. (2014). High selective H-plane TE dual mode cavity filter design by using nonresonating nodes. Microwave and Optical Technology Letters. 56(1):161-166. doi:10.1002/mop.28021.

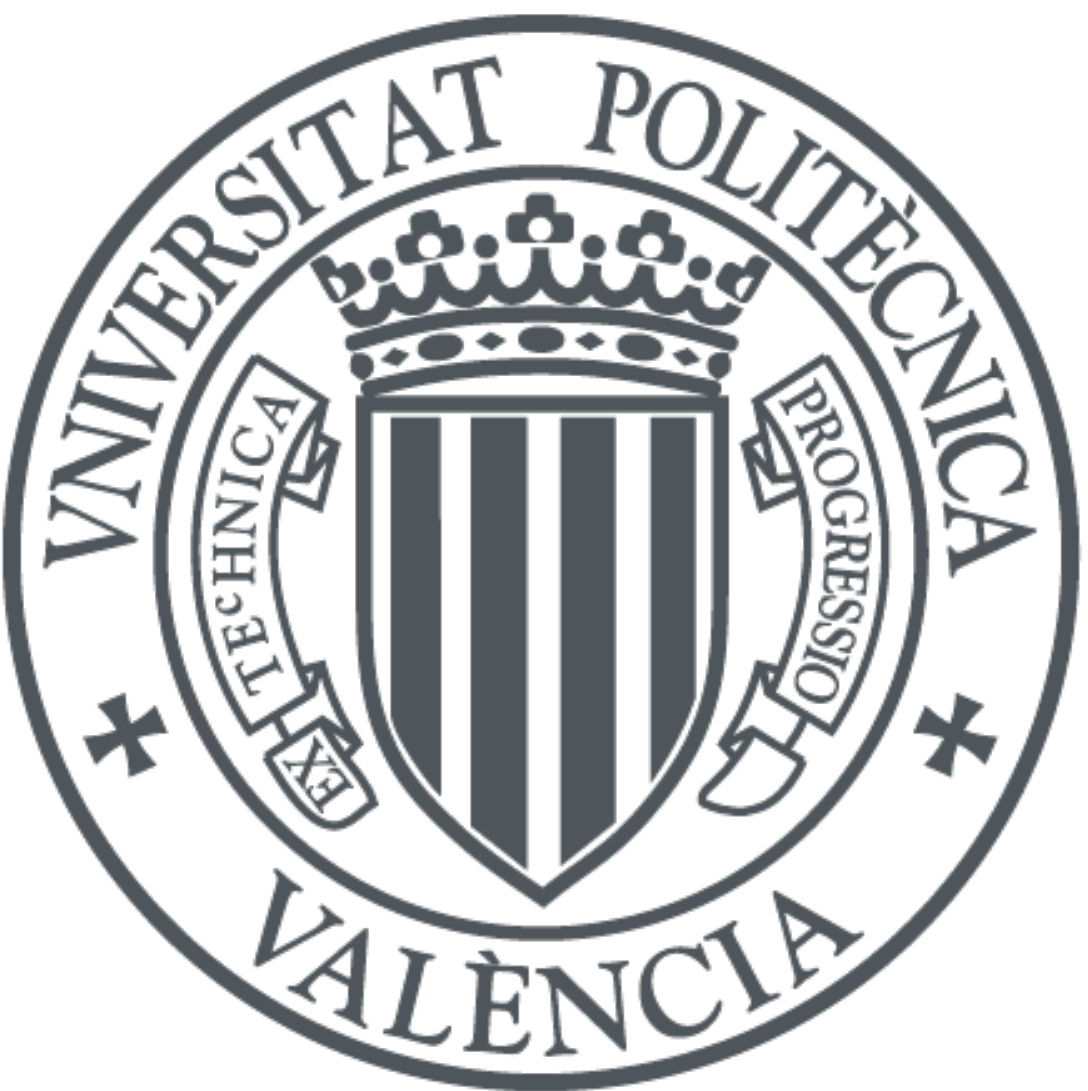

The final publication is available at

http://dx.doi.org/10.1002/mop.28021

Copyright Wiley: 12 months

Additional Information 


\title{
High Selective H-plane TE Dual Mode Cavity Filter Design by Using Nonresonating Nodes
}

\author{
B. Boukari, C. P. Vicente and S. Cogollos
}

\begin{abstract}
The design of H-plane TE dual mode cavity filters using models containing nonresonating nodes is presented. From the models a coupling matrix is derived and decomposed into submatrices, each one representing a subcircuit. The optimization and cascading of subcircuits represents a good starting point for the global optimization.
\end{abstract}

Index Terms - Coupling matrix, dual-mode filter, elliptic filter, H-plane filter, nonresonating nodes (NRNs), synthesis.

$\mathrm{M}$ ODERN microwave filters are expected to meet the increasingly stringent requirements for a variety of applications: they are required to exhibit good selectivity, low in-band insertion loss, linear phase, small size and light weight. In order to increase the selectivity, filters must present a sharp transition between pass bands and stop bands. This is achieved by generating transmission zeros. Traditionally, transmission zeros have been generated by cross-coupling non adjacent resonators [1]-[2]. The realization of coupling coefficients, in such filters, is sometimes difficult or even impossible to achieve. So, alternative solutions have been proposed by using extracted pole filters with nonresonant nodes [3]. With such filters, high selectivity can be achieved but their size remains important.

In satellite communications, dual-mode and multi-mode microwave cavity band-pass filters remain the unchallenged technology as they come very close to satisfying all these, often contradictory, requirements. Multi-mode filters exploit the coupling between different modes of a waveguide cavity in order to reduce the size and weight of the structure while offering the possibility of implementing sophisticated pseudoelliptic filtering functions.

Early developments used degenerated modes in circular or rectangular cavities realized by coupling screws, which are adjusted experimentally until the desired response is achieved [4]. Simpler elements were later introduced to replace the coupling screws [5].

Manuscript received May 1rst, 2013. This work was supported in part by Aurora Software and Testing S.L.

B. Boukari is graduated from the INRS, University of Quebec, Montreal, H5A1K6 Canada (phone: +1 514-332-8391; e-mail: bouraima.boukari@sympatico.ca).

C. P. Vicente is with Aurora Software and Testing S.L., Polytechnic University of Valencia, Valencia, 46022 Spain, (e-mail: carlos.vicente@aurorosat.es).

S. Cogollos is with the Polytechnic University of Valencia, Valencia, 46022 Spain, (e-mail: sancobo@dcom.upv.es).
More recent developments simply avoid mode degeneration, so that neither coupling elements nor screws in the center of cavities are needed. The modes inside the cavities of such filters are orthogonal. Their coupling is realized in the irises, which can be considered as non-resonating nodes. In this category of filters, we have TE/TM multi-mode cavity filters [6]-[7], which can be very compact and can exhibit very high selectivity but the insertion losses are high and their power handling is poor, because in order to excite TM modes the slots (irises) must be small.

In 2001 Guglielmi and others proposed a dual-mode microwave filter, comprising a rectangular resonator operating in two distinct $\mathrm{TE}_{\mathrm{m}, \mathrm{o}, \mathrm{n}}$ and $\mathrm{TE}_{\mathrm{p}, \mathrm{o}, \mathrm{q}}$ modes [8]-[9], which is a great challenge to the circular waveguide dual-mode, in terms of power handling and selectivity. The advantage is that the required filter structure is very simple and very suitable for high precision manufacture at low cost, thereby reducing the total cost of development and manufacture in highly significant manner. For their design, a $T E_{m, 0, n} / \mathrm{TE}_{\mathrm{p}, \mathrm{o}, \mathrm{q}}$ cavity was modeled with a circuit comprising one resonator and a second selfcoupled resonator [10]. With such an equivalent circuit, it is not possible to derive a coupling matrix.

In this paper, we focus on the design of $\mathrm{TE}_{2,0,1} / \mathrm{TE}_{1,0,2}$ dual-mode cavity filter which is the most compact among the $\mathrm{TE}_{\mathrm{m}, \mathrm{o}, \mathrm{n}}$ and $\mathrm{TE}_{\mathrm{p}, \mathrm{o}, \mathrm{q}}$ family of dual-mode cavity filters. In order to get a coupling matrix, we model a dual-mode cavity with an equivalent circuit which comprises two resonators coupled through a non-resonating node. The obtained overall coupling matrix will be decomposed into sub-matrices, each one representing the ideal response of a cavity or a group of cavities. The optimization will be performed first on the subcircuits and their connection represents a good initial point for the global optimization. In this manner, we are able for the first time, in our knowledge, to design a high selective H-plane waveguide filter with 8 poles and 4 transmission zeros, using 4 $\mathrm{TE}_{2,0,1} / \mathrm{TE}_{1,0,2}$ dual-mode cavities.

\section{THE BASIC ELEMENT}

The basic element is the $\mathrm{TE}_{201} / \mathrm{TE}_{102}$ dual-mode cavity. In order to excite $\mathrm{TE}_{\mathrm{m} 0}$ modes with $\mathrm{m}$ even, there must be an offset between the centers of irises and the cavity [8]. The greater the offset, the more important is the attenuation in the stop-band. In the extreme case, the upper walls of the cavities and irises are continuous. This case will be treated in this 
paper. Figure 1 shows the structure of the cavity. The irises are centered with regard to the input and output waveguide sections.

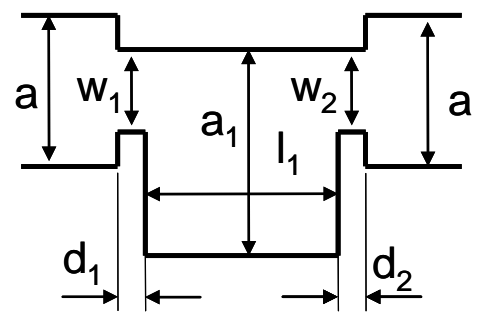

Figure 1. The structure of $\mathrm{TE}_{201} / \mathrm{TE}_{102}$ cavity

The resonance of a TE cavity mode is given by:

\section{¡Error! Marcador no}

$$
\text { definido. } f_{r}=\frac{1}{2 \sqrt{\varepsilon_{0} \mu_{0}}} \sqrt{\frac{m^{2}}{a^{2}}+\frac{n^{2}}{b^{2}}+\frac{p^{2}}{l^{2}}}
$$

If both $\mathrm{TE}_{201}$ and $\mathrm{TE}_{102}$ modes resonate at the same frequency, then we have

$$
\sqrt{\frac{4}{a^{2}}+\frac{1}{l^{2}}}=\sqrt{\frac{1}{a^{2}}+\frac{4}{l^{2}}} \Rightarrow a=l
$$

From the frequency $f_{z}$ of a prescribed transmission zero, we obtain:

$$
a=l=\frac{c_{0}}{2 f_{z}} \sqrt{5}
$$

From the study in [8], it is shown that this cavity exhibits two poles and one transmission zero. The low-pass prototype can be modeled according to the techniques developed in [10] and [11]. It contains the following four distinct types of components.

1) Resonators: These are represented by unit capacitors in parallel with frequency-independent reactances, which account for the frequency shifts in their resonant frequencies.

2) Admittance inverters. These are identical to the coupling coefficients between the nodes.

3) NRN: These are internal nodes that are connected to ground by frequency-independent reactances.

4) The input (source) and the output (load): These are normalized conductances.

A resonator that is responsible for an attenuation pole at a normalized frequency $s_{i}=j \omega_{i}$ is represented by a unit capacitor in parallel with a constant reactance $j b_{i}=-j \omega_{i}$. Such a dangling resonator is only connected to a nonresonating node (NRN).

Figure 2 shows the low-pass prototype of the cavity with a nonresonating node.

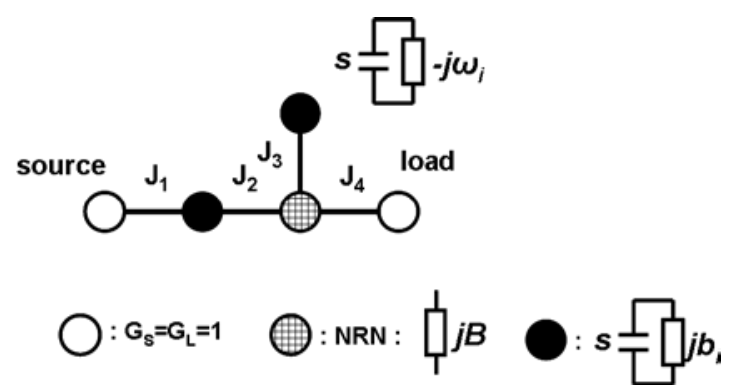

Figure 2. Low-pass prototype for a TE201/TE102 dual-mode cavity

\section{DESIGN PROCEDURE OF BAND-PASS FILTERS}

\section{A. Band-pass Filter with Two Cavities}

The structure of a band-pass filter with two $\mathrm{TE}_{201} / \mathrm{TE}_{102}$ dual-mode cavities is shown in Figure 3.

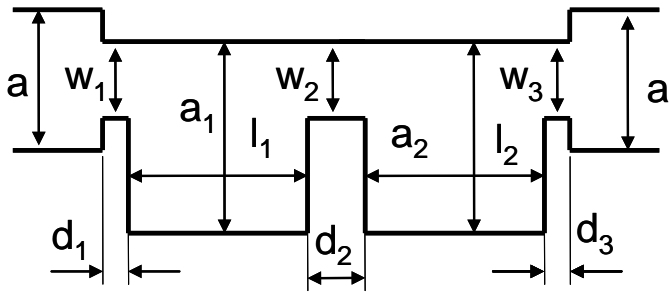

Figure 3. Topology for two-TE201/TE102 dual-mode cavity filter

Its corresponding low-pass prototype is shown in Figure 4

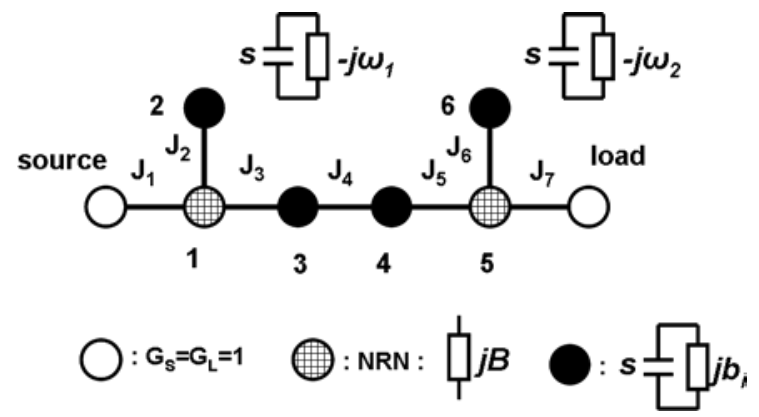

Figure 4. Low-pass prototype for two-dual-mode cavity filter in Figure 3

After the extraction of circuit elements, the coupling matrix looks like the following one: 


$$
M=\left(\begin{array}{cccccccc}
0 & J_{1} & 0 & 0 & 0 & 0 & 0 & 0 \\
J_{1} & B_{1} & J_{2} & J_{3} & 0 & 0 & 0 & 0 \\
0 & J_{2} & -\omega_{1} & 0 & 0 & 0 & 0 & 0 \\
0 & J_{3} & 0 & b_{3} & J_{4} & 0 & 0 & 0 \\
0 & 0 & 0 & J_{4} & b_{4} & J_{5} & 0 & 0 \\
0 & 0 & 0 & 0 & J_{5} & B_{5} & J_{6} & J_{7} \\
0 & 0 & 0 & 0 & 0 & J_{6} & -\omega_{2} & 0 \\
0 & 0 & 0 & 0 & 0 & J_{7} & 0 & 0
\end{array}\right)
$$

Each cavity can first be designed individually, according to the corresponding submatrix and then cascaded to the other through a quarter-wave waveguide section [7]. This represents a convenient starting point for the global optimization of the whole filter. In [7], the matrix resulting from the cascading of individual cavities is determined by optimization. In this paper, given that the extraction of elements of the whole filter presented in Figure 4 is easily done analytically, we derive the cascaded matrix from it. By cascading two cavities through nonresonating nodes and a quarter-wave waveguide section, we obtain the low-pass prototype shown in Fig. 5. All the elements remain the same, except the admittance inverters $\mathrm{J}_{\mathrm{A}}$ and $\mathrm{J}_{\mathrm{B}}$ and the two nonresonating nodes $\mathrm{L}_{1}$ and $\mathrm{S}_{2}$ with no susceptances.

If the admittance at the input of resonator 4 looking towards the load, in both circuits is denoted by $\mathrm{Y}_{4}$, then the admittance at the output of resonator 3 looking towards the load for the circuits in Figure 4 and 5 are, respectively,

$$
\begin{gathered}
Y_{3}=J_{4}{ }^{2} / Y_{4} \\
Y_{3}^{\prime}=J_{A}{ }^{2} J_{B}{ }^{2} / Y_{4}
\end{gathered}
$$

For both circuits to have the same response, we must have:

$$
Y_{3}=Y_{3}^{\prime}
$$

From this equation we obtain:

$$
J_{4}^{2}=J_{A}^{2} J_{B}^{2}
$$

This equation has an infinity number of solutions. If we set $J_{B}= \pm J_{A}$, then we have $J_{A}= \pm \sqrt{J_{4}}$ and we obtain 4 solutions:

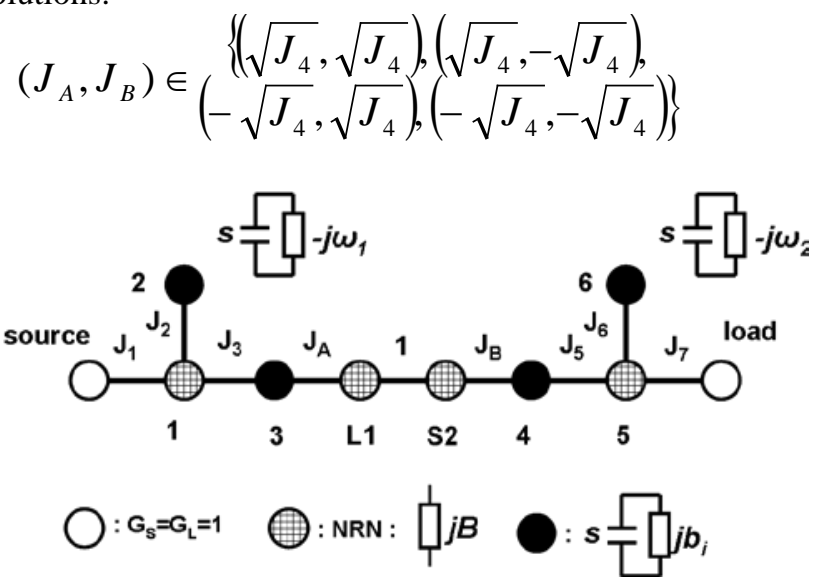

Figure 5. Low-pass prototype for two cascaded-dual-mode cavities
If we choose the first solution to obtain inductive couplings only, the matrix of the cascaded circuit becomes:

$$
M=\left(\begin{array}{cccccccccc}
0 & J_{1} & 0 & 0 & 0 & 0 & 0 & 0 & 0 & 0 \\
J_{1} & B_{1} & J_{2} & J_{3} & 0 & 0 & 0 & 0 & 0 & 0 \\
0 & J_{2} & -\omega_{1} & 0 & 0 & 0 & 0 & 0 & 0 & 0 \\
0 & J_{3} & 0 & b_{3} & \sqrt{J_{4}} & 0 & 0 & 0 & 0 & 0 \\
0 & 0 & 0 & \sqrt{J_{4}} & 0 & 1 & 0 & 0 & 0 & 0 \\
0 & 0 & 0 & 0 & 1 & 0 & \sqrt{J_{4}} & 0 & 0 & 0 \\
0 & 0 & 0 & 0 & 0 & \sqrt{J_{4}} & b_{4} & J_{5} & 0 & 0 \\
0 & 0 & 0 & 0 & 0 & 0 & J_{5} & B_{5} & J_{6} & J_{7} \\
0 & 0 & 0 & 0 & 0 & 0 & 0 & J_{6} & -\omega_{2} & 0 \\
0 & 0 & 0 & 0 & 0 & 0 & 0 & J_{7} & 0 & 0
\end{array}\right)
$$

The submatrices for the first and second cavities are, respectively,

$$
\begin{gathered}
M_{1}=\left(\begin{array}{ccccc}
0 & J_{1} & 0 & 0 & 0 \\
J_{1} & B_{1} & J_{2} & J_{3} & 0 \\
0 & J_{2} & -\omega_{1} & 0 & 0 \\
0 & J_{3} & 0 & b_{3} & \sqrt{J_{4}} \\
0 & 0 & 0 & \sqrt{J_{4}} & 0
\end{array}\right) \\
M_{2}=\left(\begin{array}{ccccc}
0 & \sqrt{J_{4}} & 0 & 0 & 0 \\
\sqrt{J_{4}} & b_{4} & J_{5} & 0 & 0 \\
0 & J_{5} & B_{5} & J_{6} & J_{7} \\
0 & 0 & J_{6} & -\omega_{2} & 0 \\
0 & 0 & J_{7} & 0 & 0
\end{array}\right)
\end{gathered}
$$

\section{B. Band-pass Filter with Four Cavities}

The structure for the band-pass filter with four $\mathrm{TE}_{201} / \mathrm{TE}_{102}$ dual-mode cavities is shown in Figure 6 and its corresponding low-pass prototype in Figure 7.

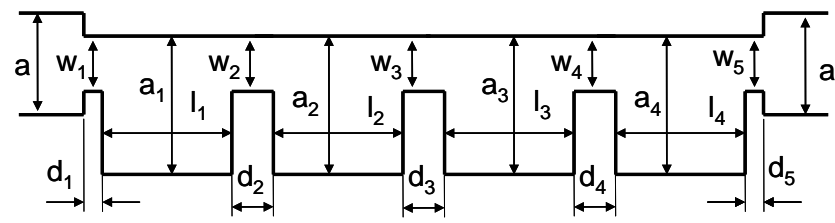

Figure 6. Topology for a dual-mode filter with four-TE201/TE102 dualmode cavities

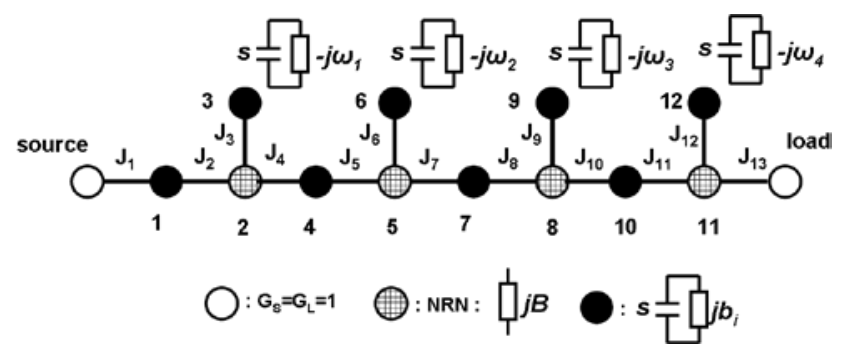

Figure 7. Low-pass prototype for four-dual-mode cavity filter in Figure 6 
Following the procedure for matrix decomposition described below, the submatrices for the four cavities are, respectively,

$$
\begin{aligned}
& M_{1}=\left(\begin{array}{ccccc}
0 & J_{1} & 0 & 0 & 0 \\
J_{1} & b_{1} & J_{2} & 0 & 0 \\
0 & J_{2} & B_{2} & J_{3} & \sqrt{J_{4}} \\
0 & 0 & J_{3} & -\omega_{1} & 0 \\
0 & 0 & \sqrt{J_{4}} & 0 & 0
\end{array}\right), \quad M_{2}=\left(\begin{array}{ccccc}
0 & \sqrt{J_{4}} & 0 & 0 & 0 \\
\sqrt{J_{4}} & b_{4} & J_{5} & 0 & 0 \\
0 & J_{5} & B_{5} & J_{6} & \sqrt{J_{7}} \\
0 & 0 & J_{6} & -\omega_{2} & 0 \\
0 & 0 & \sqrt{J_{7}} & 0 & 0
\end{array}\right) \\
& M_{3}=\left(\begin{array}{ccccc}
0 & \sqrt{J_{7}} & 0 & 0 & 0 \\
\sqrt{J_{7}} & b_{7} & J_{8} & 0 & 0 \\
0 & J_{8} & B_{8} & J_{9} & \sqrt{J_{10}} \\
0 & 0 & J_{9} & -\omega_{3} & 0 \\
0 & 0 & \sqrt{J_{10}} & 0 & 0
\end{array}\right), \quad M_{4}=\left(\begin{array}{cccccc}
0 & \sqrt{J_{10}} & 0 & 0 & 0 \\
\sqrt{J_{10}} & b_{10} & J_{11} & 0 & 0 \\
0 & J_{11} & B_{11} & J_{12} & J_{13} \\
0 & 0 & J_{12} & -\omega_{4} & 0 \\
0 & 0 & J_{13} & 0 & 0
\end{array}\right)
\end{aligned}
$$

First, the cavities are designed individually according to the above submatrices, then cascaded two by two and optimized according to the following matrices:

$$
\begin{aligned}
M_{5} & =\left(\begin{array}{cccccccc}
0 & J_{1} & 0 & 0 & 0 & 0 & 0 & 0 \\
J_{1} & b_{1} & J_{2} & 0 & 0 & 0 & 0 & 0 \\
0 & J_{2} & B_{2} & J_{3} & J_{4} & 0 & 0 & 0 \\
0 & 0 & J_{3} & -\omega_{1} & 0 & 0 & 0 & 0 \\
0 & 0 & J_{4} & 0 & b_{4} & J_{5} & 0 & 0 \\
0 & 0 & 0 & 0 & J_{5} & B_{5} & J_{6} & \sqrt{J_{7}} \\
0 & 0 & 0 & 0 & 0 & J_{6} & -\omega_{2} & 0 \\
0 & 0 & 0 & 0 & 0 & \sqrt{J}_{7} & 0 & 0
\end{array}\right), \\
M_{6} & =\left(\begin{array}{cccccccc}
0 & \sqrt{J_{7}} & 0 & 0 & 0 & 0 & 0 & 0 \\
\sqrt{J_{7}} & b_{7} & J_{8} & 0 & 0 & 0 & 0 & 0 \\
0 & J_{8} & B_{8} & J_{9} & J_{10} & 0 & 0 & 0 \\
0 & 0 & J_{9} & -\omega_{3} & 0 & 0 & 0 & 0 \\
0 & 0 & J_{10} & 0 & b_{10} & J_{11} & 0 & 0 \\
0 & 0 & 0 & 0 & J_{11} & B_{11} & J_{12} & J_{13} \\
0 & 0 & 0 & 0 & 0 & J_{12} & -\omega_{4} & \\
0 & 0 & 0 & 0 & 0 & J_{13} & 0 & 0
\end{array}\right),
\end{aligned}
$$

The whole filter is then constructed and a global optimization is performed to meet the requirements.

\section{Coupling Matrix Element Extraction}

The quasi-elliptical filter functions can be calculated through the recursive relations given in [12]. Once the filter functions are determined, the reflections coefficient $S_{11}$ and $S_{22}$ are calculated:

$$
\begin{aligned}
& S_{11}(s)=e^{j \phi_{11}} \frac{M(s)}{D(s)} \\
& S_{22}(s)=e^{j \phi_{22}} \frac{M(s)}{D(s)}
\end{aligned}
$$

From the reflection coefficients, the input and output admittances are determined

$$
\begin{gathered}
y_{\text {in }}(s)=\frac{1-S_{11}(s)}{1+S_{11}(s)}=\frac{D(s)-e^{j \phi_{11}} M(s)}{D(s)-e^{j \phi_{11}} M(s)} \\
y_{\text {out }}(s)=\frac{1-S_{22}(s)}{1+S_{22}(s)}=\frac{D(s)-e^{j \phi_{22}} M(s)}{D(s)-e^{j \phi_{22}} M(s)},
\end{gathered}
$$

From the input and output admittances, the coupling matrix elements are extracted following the procedure given in [11].

\section{DESIGN EXAMPLES}

For the analysis and optimization of the filters, a very fast full-wave electromagnetic CAD tool Fest3D has been used. The standard waveguide WR75 was used for the input and output waveguide sections ( $\mathrm{a}=19.05 \mathrm{~mm}, \mathrm{~b}=9.525 \mathrm{~mm})$.

The first example is a two-cavity fourth-order filter with two attenuation poles at normalized frequencies $s_{1}=-2.4 \mathrm{j}$ and $s_{2}=$ $2.4 \mathrm{j}$. The central frequency is $10.5 \mathrm{GHz}$ and the bandwidth is $200 \mathrm{MHz}$ with minimum return loss set to $22 \mathrm{~dB}$. The extracted circuit elements are: $\mathrm{J}_{1}=1.075, \mathrm{~J}_{2}=2.103, \mathrm{~J}_{3}=0.956$, $\mathrm{J}_{4}=0.758, \quad \mathrm{~J}_{5}=1.0, \quad \mathrm{~J}_{6}=2.2, \quad \mathrm{~J}_{7}=1.124, \quad \mathrm{~b}_{1}=2.006, \quad \mathrm{~b}_{2}=2.4$, $\mathrm{b}_{3}=0.051, \mathrm{~b}_{4}=-0.051, \mathrm{~b}_{5}=-2.195, \mathrm{~b}_{6}=-2.4$

The cavities were designed individually, according to their submatrices and then cascaded. The global optimization was then performed. The optimized filter dimensions, according to Figure 3 are as follows:

$\mathrm{a}_{1}=31.9726 \mathrm{~mm}, \mathrm{a}_{2}=30.9494 \mathrm{~mm}, \mathrm{l}_{1}=28.9567 \mathrm{~mm}, \mathrm{l}_{2}=$ $29.9116 \mathrm{~mm}, \mathrm{w}_{1}=13.399 \mathrm{~mm}, \mathrm{w}_{2}=12.9565 \mathrm{~mm}, \mathrm{w}_{3}=12.916$ $\mathrm{mm}, \mathrm{d}_{1}=6.022 \mathrm{~mm}, \mathrm{~d}_{2}=16.3549 \mathrm{~mm}, \mathrm{~d}_{3}=4.5556 \mathrm{~mm}$.

Figure 8 shows the simulated S-parameters. 


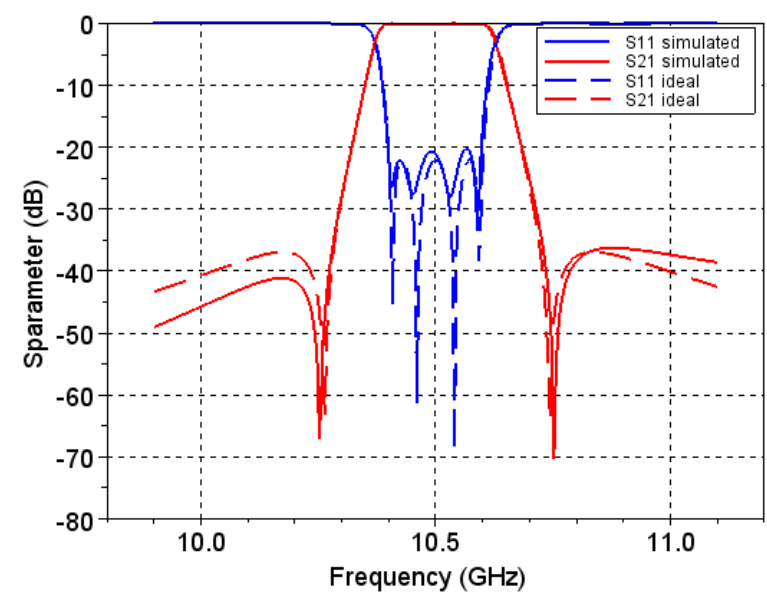

Figure 8 S-parameters for the two-cavity filter with prescribed transmission zeros at the normalized frequencies $\mathrm{s}= \pm 2.4 \mathrm{j}$

The second example is a two-cavity fourth-order filter with two attenuation poles at normalized frequencies $s_{1}=-3.2 \mathrm{j}$ and $s_{2}=-2.4 \mathrm{j}$. The central frequency is $10.5 \mathrm{GHz}$ and the bandwidth is $200 \mathrm{MHz}$ with return losses of $22 \mathrm{~dB}$. The extracted circuit elements are: $\mathrm{J}_{1}=1.075, \mathrm{~J}_{2}=3.021, \mathrm{~J}_{3}=0.956$, $\mathrm{J}_{4}=0.758, \mathrm{~J}_{5}=1.0, \mathrm{~J}_{6}=2.243, \mathrm{~J}_{7}=1.122, \mathrm{~b}_{1}=2.917, \mathrm{~b}_{2}=3.2, \mathrm{~b}_{3}=$ $-0.0095, b_{4}=-0.0875, b_{5}=2.204, b_{6}=2.4$.

The filter was designed according to the procedure developed in section II. The optimized filter dimensions, according to Figure 3 are as follows:

$\mathrm{a}_{1}=32.087 \mathrm{~mm}, \mathrm{a}_{2}=31.9729 \mathrm{~mm}, \mathrm{l}_{1}=29.7587 \mathrm{~mm}, \mathrm{l}_{2}=$ $29.9237 \mathrm{~mm}, \mathrm{w}_{1}=12.4639 \mathrm{~mm}, \mathrm{w}_{2}=11.9365 \mathrm{~mm}, \mathrm{w}_{3}=$ $12.2093 \mathrm{~mm}, \mathrm{~d}_{1}=1.5335 \mathrm{~mm}, \mathrm{~d}_{2}=9.556 \mathrm{~mm}, \mathrm{~d}_{3}=2.9072$ $\mathrm{mm}$.

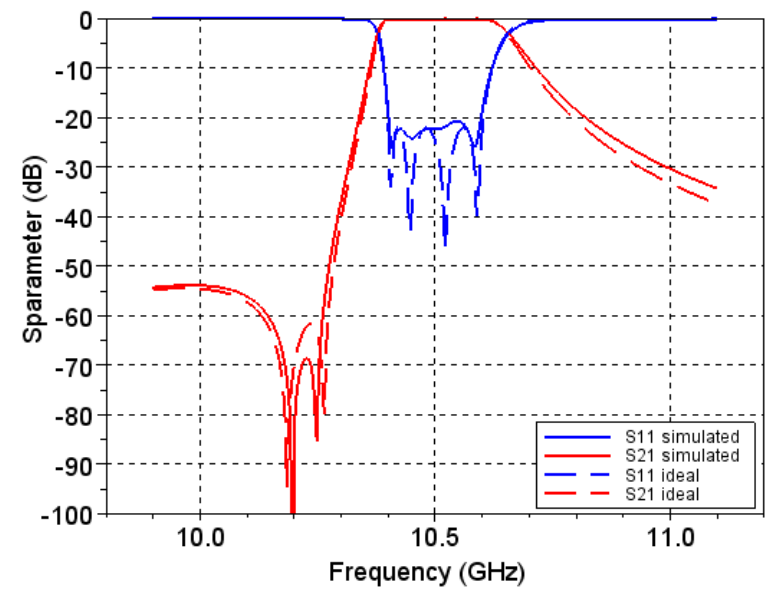

Figure 9 shows the simulated S-parameters.

Figure 9 S-parameters for the two-cavity filter with prescribed transmission zeros at the normalized frequencies $\mathrm{s}=-3.2 \mathrm{j}$ and $\mathrm{s}=-2.4 \mathrm{j}$.

The third example is a four-cavity eight-order filter with four attenuation poles at normalized frequencies $s_{1}=-2.8 \mathrm{j}, s_{2}$ $=-2.0 \mathrm{j}, s_{3}=2.0 \mathrm{j}$, and $s_{4}=2.8 \mathrm{j}$. The central frequency is 10.5 $\mathrm{GHz}$ and the bandwidth is $200 \mathrm{MHz}$ with return losses of 22
$\mathrm{dB}$. The extracted circuit elements, according to the low-pass filter-prototype presented in Figure 7, are: $\mathrm{J}_{1}=1.028, \mathrm{~J}_{2}=1.0$, $\mathrm{J}_{3}=3.047, \mathrm{~J}_{4}=0.696, \mathrm{~J}_{5}=1.0, \mathrm{~J}_{6}=3.160, \mathrm{~J}_{7}=0.992, \quad \mathrm{~J}_{8}=1.0$, $\mathrm{J}_{9}=3.160, \quad \mathrm{~J}_{10}=1.080, \quad \mathrm{~J}_{11}=1.0, \quad \mathrm{~J}_{12}=3.047, \quad, \quad \mathrm{~J}_{13}=1.204, \quad \mathrm{~b}_{1}$ $=0.273, b_{2}=3.666, b_{3}=2.80, b_{4}=0.264, b_{5}=5.943, b_{6}=2.0, b_{7}$ $=0.014, b_{8}=-5.880, b_{9}=-2.0, b_{10}=-0.192, b_{11}=-3.465, b_{12}=-$ 2.8 .

The filter was designed according to the procedure developed in section II. The optimized filter dimensions, according to Figure 6 are as follows:

$\mathrm{a}_{1}=32.037 \mathrm{~mm}, \mathrm{a}_{2}=31.898 \mathrm{~mm}, \mathrm{a}_{3}=31.202 \mathrm{~mm}, \mathrm{a}_{4}=$ $31.109 \mathrm{~mm}, \mathrm{l}_{1}=30.111 \mathrm{~mm}, \mathrm{l}_{2}=30.373 \mathrm{~mm}, \mathrm{l}_{3}=30.831 \mathrm{~mm}$, $\mathrm{l}_{4}=31.031 \mathrm{~mm}, \mathrm{w}_{1}=11.897 \mathrm{~mm}, \mathrm{w}_{2}=11.647 \mathrm{~mm}, \mathrm{w}_{3}=$ $11.686 \mathrm{~mm}, \mathrm{w}_{4}=11.718 \mathrm{~mm}, \mathrm{w}_{5}=11.345 \mathrm{~mm}, \mathrm{~d}_{1}=1.0 \mathrm{~mm}$, $\mathrm{d}_{2}=9.237 \mathrm{~mm}, \mathrm{~d}_{3}=10.068 \mathrm{~mm}, \mathrm{~d}_{4}=9.636 \mathrm{~mm}, \mathrm{~d}_{5}=1.0 \mathrm{~mm}$.

Figure 10 shows the simulated S-parameters.

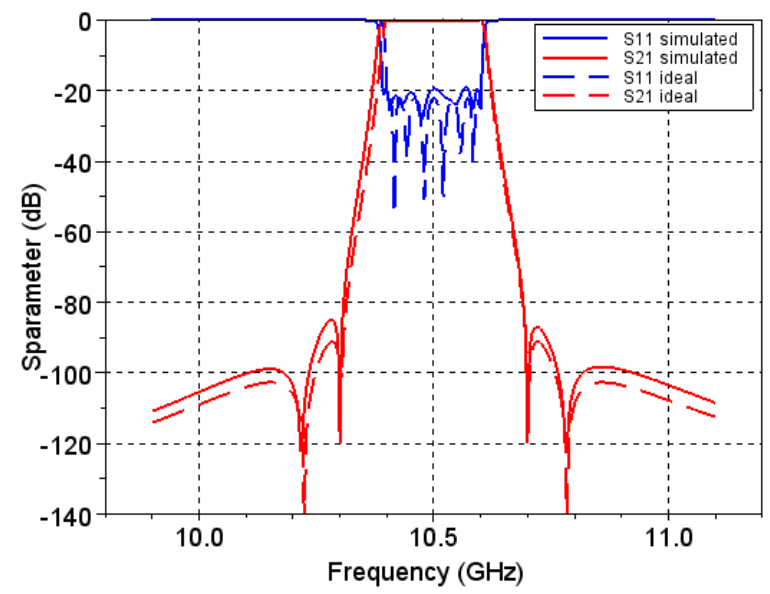

Figure $10 \mathrm{~S}$-parameters for the four-cavity filter with prescribed transmission zeros at the normalized frequencies $\mathrm{s}=-2.8 \mathrm{j}, \mathrm{s}=-2.0 \mathrm{j}, \mathrm{s}=2.0 \mathrm{j}$ and $\mathrm{s}=2.8 \mathrm{j}$

\section{CONCLUSION}

In this paper, a modular design procedure for the H-plane dual-mode cavity filters using coupling matrix decomposition derived from extracted pole models with nonresonating nodes, is presented. With this procedure a high selective eight-pole filter with four- $\mathrm{TE}_{201} / \mathrm{T}_{102}$ dual-mode cavities was designed for the first time to the authors' knowledge.

\section{ACKNOWLEDGMENT}

The authors would like to thank Aurora Software and Testing S.L. for providing the very fast full-wave CAD tool Fest3D for the analysis and optimization of the filters.

\section{REFERENCES}

[1] A. E. Atia and A. E. Williams, "New types of waveguide bandpass filters for satellite transponders," Comsat Tech. Review vol.1, No.1, pp. 20-43, Fall 1971. 
[2] Jan Kocbach and Kjetil Folgerø, "Design procedure for waveguide filters with cross-couplings,” Nera ASA, Nera Research, N-5020 Bergen, NORWAY.

[3] J. R. Montejo-Garai, J. A. Ruiz-Cruz, J. M. Rebollar, M. J. Padilla-Cruz, A. Oñoro-Navarro, and I. Hidalgo-Carpintero, "Synthesis and design of in-line $\mathrm{N}$-order filters with $\mathrm{N}$ real transmission zeros by means of extracted poles implemented in low-cost rectangular H-plane waveguide," IEEE Trans. Microwave Theory Tech., vol. 53, No.4, pp. 1636-1642, May 2005.

[4] A. E. Williams and A. E. Atia, "Dual-mode canonical waveguide filters,” IEEE Trans. Microw. Theory Tech., vol. 25, pp. 1021.1026, Dec. 1977.

[5] X. P. Liang, K. A. Zaki, and A. E. Atia, "Dual mode coupling by square corner cut in resonators and filters," IEEE Trans. Microwave Theory Tech., vol. 40, pp. 2294.2302, Dec. 1992.

[6] U. Rosenberg, S. Amari and J. Bornemann, "Inline TM110-mode filters with high design flexibility by utilizing bypass couplings of nonresonating TE10/01 modes," IEEE Trans. Microwave Theory Tech., Vol. 51, pp. 1735-1742, June 2003.

[7] S. Bastioli, C. Tomassoni, and R. Sorrentino, "A new class of waveguide dual-mode filters using TM and nonresonating modes," IEEE Trans. Microwave Theory Tech., Vol. 58, pp. 39095-3917, Dec 2010.

[8] M. Guglielmi, P. Jarry, E. Kerherve, O. Roquebrun, D. Schmitt, “A new family of all inductive Dual-Mode filters," IEEE Trans. Microwave Theory Tech., Part 1, Vol. 49, Nº10, pp. 1764-1769, Oct. 2001

[9] P. Jarry, and J. Beneat, Advanced design techniques and realizations of microwave and RF filters, John Wiley \& Sons, New Jersey 2008.

[10] S. Amari, and U. Rosenberg, "Synthesis and design of novel in-line cavity filters with one or two real transmission zeros," IEEE Trans. Microwave theory and tech., Vol. 2, No. 5, pp. 1464-1478, May 2004.

[11] S. Amari, and G. Macchiarella, "Synthesis of inline filters with arbitrarily placed attenuation poles by using non-resonating nodes," IEEE Microwave Theory and Tech., vol. 53, No. 10, pp. 3075-3081, May 2005.

[12] R. J. Cameron, "General Coupling Matrix Synthesis Methods for Chebyshev Filtering Functions," IEEE Trans. Microwave Theory Tech., vol. 47, No.4, pp. 433-442, April 1999. 